\title{
Particle imaging velocimetry experiments and lattice-Boltzmann simulations on a single sphere settling under gravity
}

\author{
A. ten Cate, ${ }^{\text {a) }}$ C. H. Nieuwstad, J. J. Derksen, and H. E. A. Van den Akker \\ Kramers Laboratorium voor Fysische Technologie, Delft University of Technology, Prins Bernhardlaan 6 , \\ 2628 BW Delft, The Netherlands
}

(Received 26 December 2001; accepted 19 August 2002; published 3 October 2002)

\begin{abstract}
A comparison is made between experiments and simulations on a single sphere settling in silicon oil in a box. Cross-correlation particle imaging velocimetry measurements were carried out at particle Reynolds numbers ranging from 1.5 to 31.9. The particle Stokes number varied from 0.2 to 4 and at bottom impact no rebound was observed. Detailed data of the flow field induced by the settling sphere were obtained, along with time series of the sphere's trajectory and velocity during acceleration, steady fall and deceleration at bottom approach. Lattice-Boltzmann simulations prove to capture the full transient behavior of both the sphere motion and the fluid motion. The experimental data were used to assess the effect of spatial resolution in the simulations over a range of 2-8 grid nodes per sphere radius. The quality of the flow field predictions depends on the Reynolds number. When the sphere is very close to the bottom of the container, lubrication theory has been applied to compensate for the lack of spatial resolution in the simulations. (C) 2002 American Institute of Physics. [DOI: 10.1063/1.1512918]
\end{abstract}

\section{INTRODUCTION}

Particle motion and particle collisions play an important role in the performance of many industrial processes involving suspension flow. For instance, in industrial crystallization, crystal-crystal collisions determine kinetic mechanisms such as agglomeration and nucleation due to crystal fracturing. Presently, we are developing a method to study suspensions under turbulent conditions. For this method, it is attempted to fully resolve the flow field around the particles and to make a direct coupling between the particle and the fluid motion. To validate the way the particles are represented in the simulation procedure, we compare experimental and numerical results on the motion of a single sphere settling in a closed box. In the experiment, the transient motion of a single sphere and its associated flow field were measured from the moment of release to a steady-state or maximum settling velocity to deceleration and rest at the bottom of the box. For this experiment, the Reynolds number, based on the steady-state settling velocity of a sphere in an infinite medium $\left(R e=\rho_{f} u_{\infty} d_{p} / \mu_{f}\right)$ was varied between 1.5 and 32. This range of Reynolds numbers was chosen because it corresponds to the range of Reynolds numbers encountered in our sample crystallization process for production of ammonium sulfate crystals. ${ }^{1}$

A particle settling towards a wall has been studied previously by Brenner, ${ }^{2}$ who derived an analytical solution in the creeping flow regime. However, at the Reynolds numbers studied, the particle is well out of this regime. Recent experimental work in this field has been presented by several authors, ${ }^{3-6}$ who studied the wall approach and rebound, to

a) Telephone: +13-15-2781400; fax: +13-15-2782838; electronic mail: A.tenCate@tnw.tudelft.nl obtain restitution coefficients for submerged particles colliding with a wall. The parameter that determines rebound is the Stokes number $\left(S t=1 / 9 R e \rho_{p} / \rho_{f}\right)$. Gondret et al. ${ }^{3}$ demonstrated that the critical Stokes number above which rebound occurs is approximately 10. In our experiments, the Stokes number was varied between 0.19 and 4.13 and no rebound was observed.

We chose to perform the experiment in a closed container for a number of reasons. First, the box width to particle diameter ratio was kept relatively small, to avoid assumptions regarding the domain size and external boundary conditions in the simulations. Thus, the influence of the container walls on the particle motion is contained both in the experiment and in the numerical simulation. Second, the experiment is transient and has a limited time-span. This has the advantage that the transient character of the simulations can be assessed throughout the different stages of acceleration, steady fall and deceleration at bottom approach. The first objective of this paper is to present our experimental data on a settling sphere in a confined geometry. The data set consists of the velocity field of the fluid surrounding the settling sphere and the trajectory (i.e., position as a function of time). The velocity field has been measured using cross correlation particle image velocimetry ${ }^{7}$ (PIV). The PIV experiment is described in the next section. The second objective is to present our approach to the simulation of freely moving particles based on the lattice-Boltzmann method. This method was chosen because it provides a robust numerical scheme that can efficiently treat the complex geometry of freely moving particles. A further advantage of this method is that it can be parallelized at high computational efficiency. The use of the lattice-Boltzmann scheme for simulation of suspensions has been proposed by Ladd, 8,9 who also presented validation of his method. A number of 


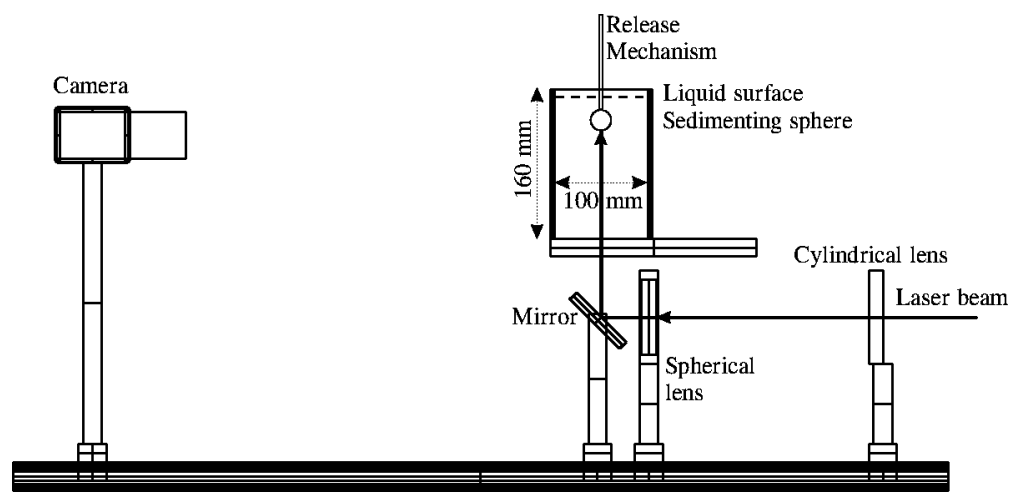

FIG. 1. Experimental setup for the PIV measurement of a single settling particle in a confined geometry.

other authors ${ }^{10-12}$ have used the lattice-Boltzmann method to simulate suspended particles. Recent developments on direct simulation of suspensions can also be found in Koch and Hill. $^{13}$

Our approach differs from the method of these authors in two ways. First, we use a scheme for solving the latticeBoltzmann equation, based on the work of Eggels and Somers. ${ }^{14}$ This scheme explicitly treats the higher order terms in the lattice-Boltzmann equation which improves the stability of the numerical scheme and allows for the use of a low kinematic viscosity. Second, we use a fundamentally different method for implementation of the moving no-slip boundary at the surface of the solid particle. In our approach, we apply the adaptive force-field technique that has been used in the work of Derksen and Van den Akker ${ }^{15}$ to implement both the rotating impeller and the steady vessel walls in the numerical study of the turbulent flow in a stirred vessel. To our knowledge this approach has not been applied previously for simulating freely moving particles. However, based on the good results obtained in previous studies, ${ }^{15,16}$ we had confidence that this approach could also be used to simulate freely moving particles at a relatively low spatial resolution per particle, bearing in mind that the method needs to be applied to simulate many particles $\left(O\left(10^{3}\right)\right)$ in a turbulent flow.

Therefore, our final objective is to assess and benchmark our numerical method with the flow cases considered in this paper. In Sec. III, a number of specific details of the setup of our particle simulations are discussed. A comparison between the experimental and numerical results is made in Sec. IV.

\section{EXPERIMENTAL SETUP}

The objective of the experiment was to accurately measure both the trajectory (i.e., the position as a function of time) and the associated flow field of a settling sphere from its moment of release until rest at the bottom of a vessel, where the ratio of the vessel dimensions to sphere radius was kept relatively small, such that the full flow field could be simulated under identical conditions.

As the settling sphere, a precision Nylon bearing with a diameter $d_{p}=15 \mathrm{~mm}$ and density $\rho_{p}=1120 \mathrm{~kg} / \mathrm{m}^{3}$ was selected. The container dimensions were chosen as depth $\times$ width $\times$ height $=100 \times 100 \times 160 \mathrm{~mm}$ (see Fig. 1). Upon start of an experiment, the sphere was released while simultaneously triggering the camera system to start filming. The sphere was hanging $120 \mathrm{~mm}$ from the bottom of the tank at the capillary tip of a Pasteur pipette that was connected to a vacuum system. The sphere was released by abruptly opening an electronic valve, thus disconnecting the vacuum from the pipette.

Various types of silicon oil were used as the working fluid, because of their good optical accessibility and weak temperature dependency of the viscosity. The Reynolds number of the settling sphere was based on the sedimentation velocity $u_{\infty}$ of a sphere in an infinite medium. To determine $u_{\infty}$, a relation for the drag coefficient due to Abraham $^{17}$ was used:

$$
C_{d}=\frac{24}{(9.06)^{2}}\left(\frac{9.06}{\sqrt{\mathrm{Re}}}+1\right)^{2} .
$$

The four experimental cases are defined in Table I.

The flow field was visualized with seeding particles illuminated with a (laser) light sheet. As the light source, a Spectra-Physics 4W Argon-ion laser (2016-05) operating in all lines mode was used. A cylindrical and a spherical lens converted the laser beam into a sheet of $190 \mu \mathrm{m}$ thickness. The laser sheet entered the vessel via the bottom. Neutrally buoyant hollow glass spheres approximately $10 \mu \mathrm{m}$ in size were used as seeding particles. A continuous camera with a

TABLE I. Setup of the sedimentation experiments.

\begin{tabular}{lccccccc}
\hline \hline Case number & $\begin{array}{c}\rho_{f} \\
{\left[\mathrm{~kg} / \mathrm{m}^{3}\right]}\end{array}$ & $\begin{array}{c}\mu_{f} \\
{\left[\mathrm{Ns} / \mathrm{m}^{2}\right]}\end{array}$ & $\begin{array}{c}u_{\infty} \\
{[\mathrm{m} / \mathrm{s}]}\end{array}$ & $\begin{array}{c}\text { Re } \\
{[-]}\end{array}$ & $\begin{array}{c}S t \\
{[-]}\end{array}$ & $\begin{array}{c}\text { Camera frequency } \\
{\left[\mathrm{s}^{-1}\right]}\end{array}$ & $\begin{array}{c}\text { Resolution } \\
{[-]}\end{array}$ \\
\hline Case E 1 & 970 & 373 & 0.038 & 1.5 & 0.19 & 60 & low \\
Case E 2 & 965 & 212 & 0.060 & 4.1 & 0.53 & 100 & low \\
Case E 3 & 962 & 113 & 0.091 & 11.6 & 1.50 & 170 & high \\
Case E 4 & 960 & 58 & 0.128 & 31.9 & 4.13 & 248 & high \\
\hline \hline
\end{tabular}




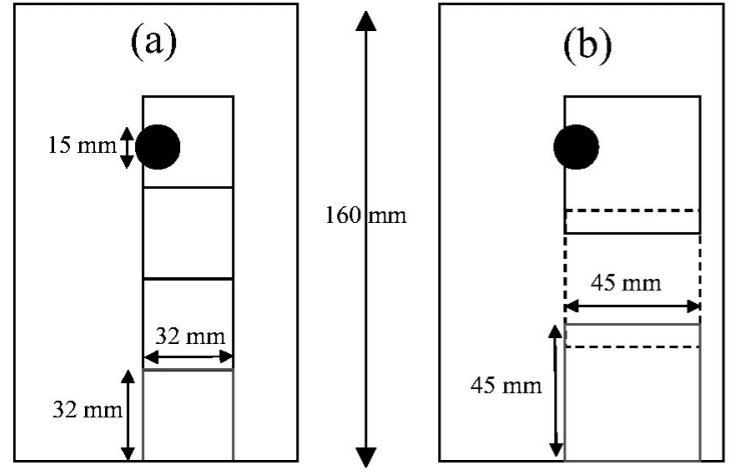

FIG. 2. Measurement positions at high (a) and low (b) resolution.

frame rate up to $250 \mathrm{~Hz}$ and an array size of 512 $\times 512$ pixels was used to record the experiment. The flow field was measured on a grid of interrogation areas (IA's). An IA typically contained $32 \times 32$ pixels. The fluid velocity was determined in each interrogation area by estimating the displacement of the seeding particles between two consecutive frames through cross correlation. ${ }^{18}$

The desired spatial resolution and the maximum camera frame rate set a restriction to the maximum fluid velocity that can be accurately measured, as between two frames the tracer particles are not allowed to shift more than 1/4 part of the linear size of an interrogation area. ${ }^{7}$ This limits the maximum sedimentation velocity of the sphere, which can be taken as a measure for the maximum fluid velocity during an experiment. The required resolution depends on the Reynolds number of the flow, because at higher Reynolds numbers, the structures in the flow become smaller. The flow was measured at either of the two resolutions given in Fig. 2. At the low resolution [Fig. 2(b)], when the array size is chosen to map three sphere diameters, the maximum allowed sphere velocity is $0.18 \mathrm{~m} / \mathrm{s}$, which is larger than any of the settling velocities of the Nylon sphere as given in Table I. At the high resolution [Fig. 2(a)], the maximum allowed velocity is 0.13 $\mathrm{m} / \mathrm{s}$, which is close to the sedimentation velocity of case $E 4$. However, the sphere is expected to move at a velocity that is lower than $u_{\infty}$ due to hindrance from the container walls. Based on the 1/4 part displacement rule, the camera frame rate was adjusted for each experiment.

To capture the full trajectory of the particle, three overlapping fields of view (FOV) were used at low resolution (cases $E 1$ and $E 2$ ) while the measurements at high resolution (cases $E 3$ and $E 4$ ) were done in four FOV's (see Fig.
2). A raw image is given in Fig. 3(a). As can be seen, the leading side of the sphere was made dark to prevent overradiation due to reflections at the sphere surface. In each frame, the sphere position had to be determined accurately for a good interpretation of the flow field. Because the laser sheet enters from the bottom and is blocked by the sedimenting sphere, no fluid velocities could be measured behind the sphere [Fig. 4(a)]. The sphere's position was determined from the colored top of the sphere. The motion blur was removed from the sphere by using an edge-preserving $\mathrm{Ku}$ wahara filter ${ }^{19}$ [Fig. 3(b)] and after having applied a threshold [Fig. 3(c)], the $y$ position of the sphere was determined at pixel accuracy [Fig. 3(d)]. The resulting sphere trajectories and velocities are given in Fig. 5.

A raw vector image of the flow at a particle Reynolds number of 1.5 is given in Fig. 4(a). Interrogation was done with IA's of $32 \times 32$ pixels with a $50 \%$ overlap, resulting in a total of 961 vectors per image. After determination of the sphere position, the vectors inside and behind the sphere were removed from the image and (for reference) a sphere was placed in the figure, as can be seen in Fig. 4(b). In this figure, the maximum velocities are found closely underneath the sphere where tracer particle displacements of approximately 7 pixels were found. Velocities far away from the sphere become very low, which gives particle displacements smaller than 0.5 pixel. Sub-pixel displacements were estimated using a Gaussian peak fit estimator. ${ }^{18}$ The accuracy is approximately 0.1 pixel for displacements larger than 0.5 pixel, leading to a relative error of $2 \%$ for the highest velocities, whereas the relative error is approximately $17 \%$ for particle displacements less than 0.5 pixel. After removal of the vectors inside and behind the sphere, approximately $10 \mathrm{spu}-$ rious vectors remain per frame. This is about $1 \%$ of the total amount of vectors which is low compared to a typical value of $5 \%$ encountered in turbulent flow fields. ${ }^{20}$ This is due to the flow being laminar and virtually two-dimensional in the center plane of the sphere, which results in practically no out of plane motion. To test the reproducibility of the experiment, all measurements were done twice. In Figs. 5(c) and 5(d), the trajectories and sedimentation velocities have been plotted for case $E 1$. The two trajectories practically coincide. The measurements at higher Reynolds numbers give comparable results. The flow fields around the sphere of case $E 1$ are presented in Fig. 4 to demonstrate that the PIV measurements yields an accurately reproducible result here as well.
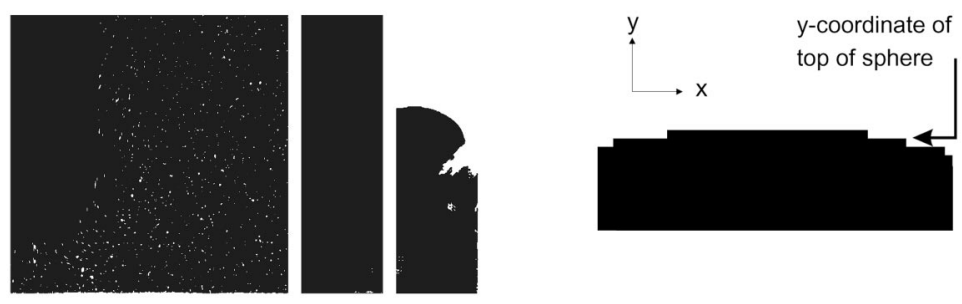

(a) (b)

(c)
FIG. 3. Processing steps in detection of sphere position. (a) Raw PIV-recording with sphere. (b) Part of recording after a Kuwahara-filter has been applied. (c) Result of thresholding image (b). (d) Magnification of the rear side of the sphere. 


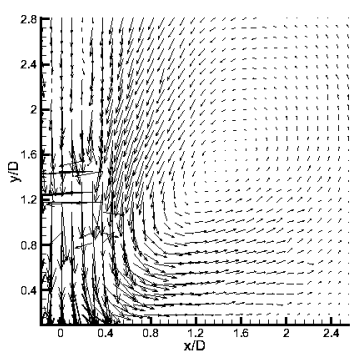

(a)

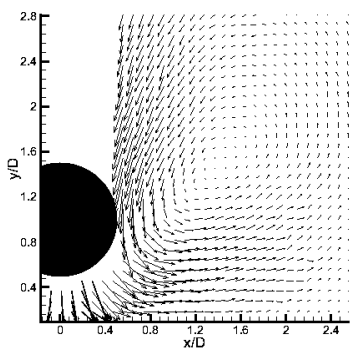

(b)

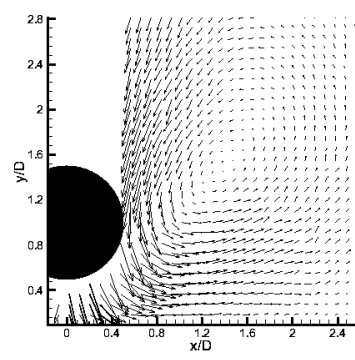

(c)
FIG. 4. Measurement of the flow field at $R e=1.5(E 1)$ at a dimensionless gap height of $h / d_{p}=0.5$. Raw flow field (a) and flow field after removal of the vectors at and behind the sphere (b) with a second measurement at the same Reynolds number and sphere position (c).

\section{SIMULATION}

For computational simulation of the sedimentation experiment, a lattice-Boltzmann scheme $^{21}$ was used. This scheme exploits a microscopic model for fluid motion on a uniform cubic lattice. Fluid masses propagate on the lattice. Collision rules that conserve mass and momentum guarantee that, in the limit of low Mach numbers, both the continuity equation and the Navier-Stokes equations for incompressible fluid flow are satisfied.

In this section, a number of issues will be addressed that had to be solved to make our simulation method applicable to freely moving particles. First, we explain the implementation of the no-slip boundary condition at the particle surface and then we discuss the issues of (i) treatment of the inertia of the internal fluid that may affect the particle motion in a nonphysical manner and (ii) the nonphysical dependency of the drag-force on the viscosity. As a consequence of this dependency, if one wants to perform accurate dynamic simulations of particles with a radius of a limited number of grid nodes, (iii) a separate calibration procedure for the particle radius is required. This calibration and scaling procedure of the simulations is discussed and finally (iv) the application of an additional lubrication force is presented, which is required when the distance between the particle and the wall has reduced to less than one grid spacing.

\section{A. Boundary conditions}

The implementation of (moving) boundary conditions in the lattice-Boltzmann framework has received considerable attention in recent years and constitutes a field of ongoing research. $^{22-25}$ Most of the methods presented in these references are adaptations of the bounce back rule for boundary

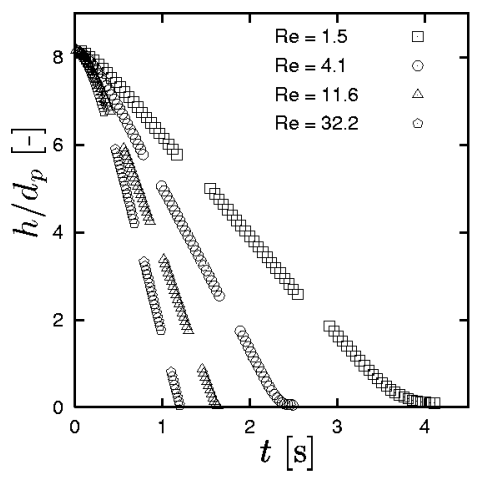

(a)

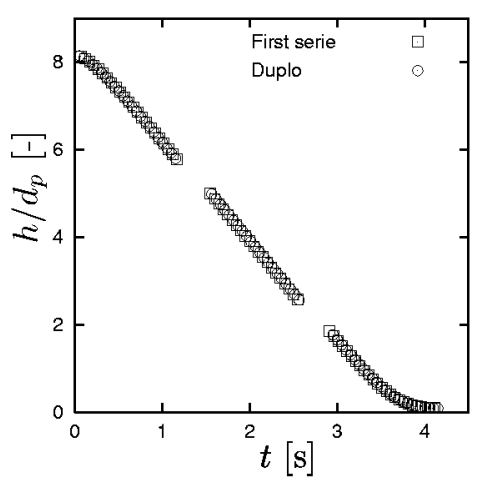

(c)

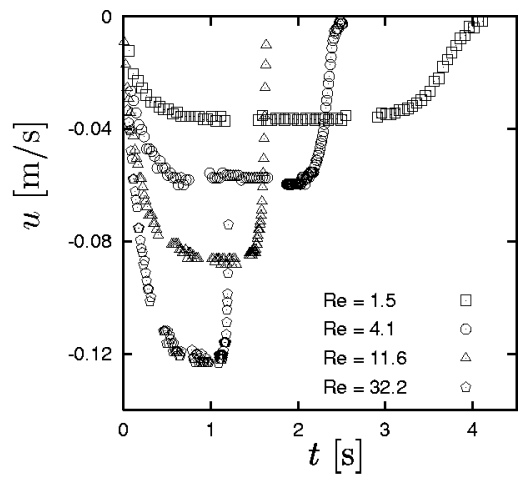

(b)

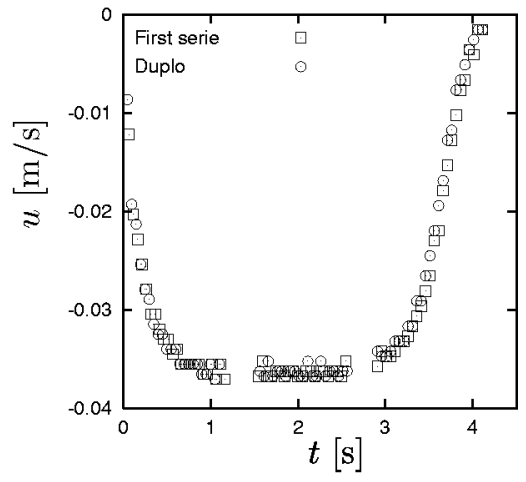

(d)
FIG. 5. Experimental data on sphere trajectory (a) and sedimentation velocity (b) at the four measured Reynolds numbers and comparison of measurement duplication of trajectory (c) and sedimentation velocity (d) at $\operatorname{Re}=1.5$. 
conditions, as explained by Ladd. ${ }^{8}$ In the standard application of the bounce back rule a link between two grid nodes that crosses a no-slip boundary is cut and mass that propagates from the flow domain towards the solid boundary is reflected along the link of its origin. One drawback of this approach is that in the straightforward implementation according to $\operatorname{Ladd}^{8}$ curved solid objects are approximated by staircase shaped objects.

In the current application the boundary of the solid sphere is implemented via the so-called adaptive force-field technique, similar to Derksen and Van den Akker. ${ }^{15}$ The scheme is based on a method for implementation of no-slip boundary conditions in spectral simulations. ${ }^{26}$ The method locally forces the fluid velocity near the boundary and interferes with the flow field not at the mesoscopic level of the propagating fluid mass but at the macroscopic level of the fluid velocities. An advantage of the method is that implementation of curved boundary conditions is done via an interpolation-extrapolation scheme which produces a smooth surface in stead of a sharply cut staircase shaped surface. Specifically for moving surfaces this is an advantageous feature since it reduces unphysical shape changes of the surface due to its motion with respect to the grid.

The sphere surface is represented by a set of $M$ control points, placed at the sphere's surface, evenly spaced at a resolution slightly higher than the grid spacing. At each control point, the local fluid velocity is forced to the surface velocity of the sphere. The steps in the procedure are as follows: First, the fluid velocity at a control point is determined via first-order Lagrange interpolation from the velocity at the surrounding grid nodes. Then, the deviation between the desired surface velocity $\boldsymbol{u}_{i}$ at control point $i$ and the interpolated fluid velocity $\boldsymbol{u}$ is used to determine the force that needs to be applied to the fluid to better approach the local surface velocity. The surface velocity is given by the translational and rotational velocity components

$$
\boldsymbol{u}_{i}=\boldsymbol{u}_{p}+\boldsymbol{\Omega}_{p} \times \boldsymbol{r}_{i},
$$

where $\boldsymbol{r}_{i}$ is the position of the control point relative to the center-of-mass of the sphere $\left(\boldsymbol{r}_{i}=\boldsymbol{x}_{i}-\boldsymbol{x}_{c}\right)$. The deviation of the fluid velocity at a control point $i$ is calculated by

$$
\boldsymbol{d}_{i}=\boldsymbol{u}_{i}-\sum_{j} I\left(\boldsymbol{r}_{i j}\right) \boldsymbol{u}_{j}
$$

where $\boldsymbol{u}_{j}$ is the fluid velocity at grid node $j$ and $I\left(\boldsymbol{r}_{i j}\right)$ is the set of interpolation coefficients, which is a function of the relative distances $\boldsymbol{r}_{i j}$ between the surrounding lattice nodes $j$ and the control point $i$. The coefficients that were used for interpolation were also used for projection of the force from the control point onto the surrounding grid nodes, where the forces $\left(\boldsymbol{F}_{j}\right)$ are updated with a relaxation scheme

$$
\boldsymbol{F}_{j}(\mathbf{x}, t)=\alpha \boldsymbol{F}_{j}(\mathbf{x}, t-1)+\beta I\left(\boldsymbol{r}_{i j}\right) \rho \boldsymbol{d}(t),
$$

where $t$ indicates the time step. We move to the next control point and the procedure is repeated until all $M$ control points have been updated. If desired, this procedure can be iterated.

The scheme of Eq. (4) that imposes locally the desired boundary condition is a control scheme that is characterized by the relaxation parameters $\alpha$ and $\beta$. The scheme adjusts the imposed body force $\boldsymbol{F}_{j}(\mathbf{x}, t)$ proportional to the deviation of the local velocity. The dynamic action, accuracy, and stability of the control scheme is determined by the relaxation parameters and the topology of the control points $\boldsymbol{x}_{i}$. The parameters $\alpha$ and $\beta$ were obtained by trial and error as 0.95 and 1.8 , respectively.

After having updated the forces at the grid nodes, we calculate the hydrodynamic force and torque on the sphere according to

$$
\begin{aligned}
& \boldsymbol{F}_{p}=\sum_{j} \boldsymbol{F}_{j}(\mathbf{x}, t), \\
& \boldsymbol{T}_{p}=\sum_{j} \mathbf{r}_{j} \times \boldsymbol{F}_{j}(\mathbf{x}, t),
\end{aligned}
$$

where $r_{j}$ is the relative distance between the grid node $j$ and the center of mass of the particle. The summation is over all boundary nodes in both the external and internal regions at the sphere's surface. The force and torque are used to integrate the equations of motion of the sphere. This is done by using an Euler forward integration scheme where the forces are averaged over two time steps to suppress unphysical fluctuations.

\section{B. Internal mass}

The boundary condition scheme requires the objects to have internal fluid. An advantage of this is that when a node shifts from the inside of an object into the exterior, it already contains fluid mass and the state of this node does not need to be changed. For the same reason, $\operatorname{Ladd}^{9}$ also has internal mass in his particles. A drawback of keeping internal mass is that its inertia affects the motion of the sphere via a contribution to $\boldsymbol{F}_{p}$ [see Eq. (5)]. Different approaches have been proposed to solve this problem. Ladd ${ }^{9}$ suggests to integrate the equation of motion with a corrected particle mass. Although this is a fair approximation for systems with large solid-fluid density ratios, for solid particles in liquid with a density ratio typically between 1 and 2 , numerical instabilities can occur when integrating the equation of motion. Other authors ${ }^{12,27,28}$ have proposed methods to remove the internal mass, but this cannot be done for the adaptive forcefield technique. $\mathrm{Qi}{ }^{11}$ proposes to compensate the hydrodynamic force $\boldsymbol{F}_{p}$ for contribution from nodes entering or leaving the interior of a particle, which is comparable to our approach.

The force applied to the fluid nodes influences the fluid on both the inside and the outside of the sphere. Hence, the total force that acts on the sphere, as calculated with Eq. (5), is the sum of the internal and external components of the force

$$
\boldsymbol{F}_{p, \text { tot }}=\boldsymbol{F}_{p, \text { int }}+\boldsymbol{F}_{p, \text { ext }} .
$$

When integrating the equation of motion, the only physical contribution to the sphere's motion comes from the external flow field. To determine the external contribution $\boldsymbol{F}_{p \text {, ext }}$, we calculate the change of momentum of the internal fluid between two successive time steps 


$$
\boldsymbol{F}(t)_{p, \text { int }}=\iiint_{V_{\text {sphere }}} \rho \boldsymbol{u}_{\text {int }}(x, t)-\rho \boldsymbol{u}_{\text {int }}(x, t-1) d V,
$$

and subtract this from the total force $\boldsymbol{F}_{p \text {,tot }}$. When using this approach the correct physical behavior is obtained, which allows us to simulate particle motion at a density ratio as low as 1.15 , as is demonstrated in Sec. IV. A similar correction procedure is applied for the torque.

\section{Low Reynolds number calibration}

Results presented by Ladd ${ }^{9}$ indicated that boundary conditions in lattice-Boltzmann schemes based on the bounce back rule suffer from a nonphysical dependency of the resulting drag force on the kinematic viscosity. A study by Rohde et $\mathrm{al}^{25}$ indicated that also more advanced boundary condition methods that are based on the bounce back rule still exhibit this behavior. A detailed analysis of this behavior is given by $\mathrm{He}$ et al. ${ }^{29}$ who demonstrate that in latticeBoltzmann methods, the exact position at which the no-slip condition is obtained is a function of the kinematic viscosity. Although there is a fundamental difference between the bounce back boundary condition and our approach, this nonphysical dependency is also observed in our current approach.

An explanation for the fact that this behavior is also observed in our simulations may be that due to the interpolation and extrapolation procedure, the sphere's surface is smeared out and the fluid experiences a sphere that is slightly bigger than the sphere on which the $M$ control points lie. The result of this effect is that the drag force obtained from the simulation is larger than the force that would correspond to the sphere's given input radius.

To compensate for this effect, $\operatorname{Ladd}^{9}$ proposed a procedure for estimating the effective sphere radius (hereafter called hydrodynamic radius). Ladd demonstrated that the hydrodynamic radius varied with viscosity as approximately one grid node. The calibration procedure is based on an analytic expression of Hasimoto ${ }^{30}$ for the drag force on a fixed sphere in a periodic array of spheres in the creeping flow regime

$$
\begin{aligned}
& \frac{6 \pi \mu r_{p} U_{v}}{F_{p}}=1.0-1.7601 C_{\tau}^{1 / 3}+C_{\tau}-1.5593 C_{\tau}^{2}, \\
& C_{\tau}=\frac{4 \pi r_{p}^{3}}{3 L^{3}}
\end{aligned}
$$

where $r_{p}$ is the sphere radius, $L$ indicates the size of the unit cell, and $U_{v}$ is the volumetrically averaged fluid velocity across the periodic cell. For a given fluid velocity and drag force, Eq. (9) is solved to calculate the hydrodynamic radius.

In our simulations we use a similar calibration procedure as proposed by Ladd. We want to stress here that this calibration procedure is performed independent of the experimental conditions or results. The sole purpose of this procedure is to determined the equivalent particle diameter, given a certain viscosity. A sphere is placed in the center of a fully periodic cell and the fluid is set into motion via a pressure gradient, such that the Reynolds number remains small. The hydrodynamic radius is determined as the average of the radius at 20 sphere positions, which were taken parallel to the flow because the settling sphere also moves along a single axis. One can ask if this low Reynolds number calibration procedure is allowed when it is our objective to simulate the transient motion of a sphere moving at nonzero Reynolds numbers. Therefore, in Sec. IV D the sensitivity of the simulations to the hydrodynamic radius is investigated.

\section{Scaling}

When setting up a simulation of the sedimentation experiment, the scaling of mass, length, and time needs to be determined. With respect to mass, only the ratio of fluid and solid density enters the equations of motion of the system. At a constant ratio, the actual values can be chosen arbitrarily without influencing the simulation result. Their numerical values were set identical to the experimental values. Length and time are scaled by using the low Reynolds number calibration procedure. A first estimate for the length scale is based on the input radius of the sphere. A first estimate for the time scale is then determined by setting $u_{\infty}$ to $0.01 \mathrm{lu} / \mathrm{ts}$ [in lattice-Boltzmann simulations, $u_{\max } \ll c_{s}$ (the speed of sound, $c_{s}=\frac{1}{2} \sqrt{2}$ ) is required to assure incompressible flow conditions]. With these first estimates, all parameters are scaled from the physical experiment into lattice units. Based on this first scaling, a calibration simulation is carried out to determine the hydrodynamic radius. Finally, in the sedimentation simulations, length is scaled on the basis of the hydrodynamic radius and time is scaled via the kinematic viscosity. In Sec. IV, calibration results of input radii between 2 and 8 lattice units will be presented, and the sensitivity of the simulations to the hydrodynamic radius will be discussed.

\section{E. Sub-grid lubrication force}

When simulating a sphere approaching a fixed wall, at some moment in time the grid lacks resolution to resolve the flow in the gap between the sphere and the wall. The repulsive forces that occur due to the squeezing motion of the fluid in the gap can no longer be computed accurately. This problem was noticed by Ladd, ${ }^{31}$ who proposed to include an explicit expression for the leading order lubrication forces, calculated with lubrication theory. ${ }^{32,33}$ In our simulations, when the gap has become smaller than $\Delta_{0}$ (set to 1 grid spacing), the force acting on the sphere due to the lubrication in the unresolved gap is calculated explicitly. The additional lubrication force at gap distance $h$ is calculated with

$$
\boldsymbol{F}_{w}=-6 \pi \mu r_{p} u_{\perp}\left(\frac{r_{p}}{h}-\frac{r_{p}}{\Delta_{0}}\right),
$$

where $h$ is the gap between the wall and the sphere and $u_{\perp}$ is the velocity component of the sphere perpendicular to the wall. In the following section, the validity of this approach will be tested by comparing simulation results with experimental data. 
TABLE II. Overview of sedimentation simulations. The table contains the input radius and calibrated radius of the sphere, with corresponding length (L.S.) and time (T.S.) scaling factors. L.F. indicates the use of lubrication theory at bottom approach. $u_{\max } / u_{\infty}$ is the ratio of the maximum sedimentation velocity and the theoretical steady-state velocity of a freely moving sphere in an infinite medium. At the bottom of the table, the experimentally obtained velocity ratio is included for comparison.

\begin{tabular}{|c|c|c|c|c|c|c|c|}
\hline Case & $\begin{array}{c}r_{0} \\
{[1 \mathrm{u}]}\end{array}$ & $\begin{array}{c}r_{h} \\
{[\mathrm{lu}]}\end{array}$ & $\begin{array}{l}R e \\
{[-]}\end{array}$ & $\begin{array}{c}\text { L.S. } \\
10^{-3} \mathrm{~m} / \mathrm{lu}\end{array}$ & $\begin{array}{c}\text { T.S. } \\
10^{-4} \mathrm{~s} / \mathrm{ts}\end{array}$ & $\begin{array}{l}\text { L.F. } \\
{[-]}\end{array}$ & $\begin{array}{c}u_{\max } / u_{\infty} \\
{[-]}\end{array}$ \\
\hline S 1 & 4 & 4.487 & 1.5 & 1.671 & 3.891 & $\ldots$ & 0.894 \\
\hline S 2 & 4 & 4.562 & 4.1 & 1.645 & 2.410 & $\ldots$ & 0.950 \\
\hline S 3 & 4 & 4.657 & 11.6 & 1.610 & 1.526 & $\ldots$ & 0.955 \\
\hline S 4 & 4 & 4.810 & 31.9 & 1.559 & 1.010 & $\cdots$ & 0.947 \\
\hline S 5 & 8 & 8.084 & 1.5 & 0.928 & 2.398 & $\ldots$ & 0.857 \\
\hline S 6 & 8 & 8.689 & 31.9 & 0.863 & 0.619 & $\ldots$ & 0.947 \\
\hline S 7 & 2 & 2.698 & 1.5 & 2.780 & 5.381 & $\ldots$ & 0.889 \\
\hline S 8 & 2 & 3.003 & 31.9 & 2.497 & 1.295 & $\ldots$ & 0.921 \\
\hline S 9 & 4 & 4.000 & 1.5 & 1.875 & 4.897 & $\ldots$ & 0.768 \\
\hline S 10 & 4 & 4.400 & 1.5 & 1.705 & 4.047 & $\ldots$ & 0.871 \\
\hline S 11 & 4 & 4.600 & 1.5 & 1.630 & 3.703 & $\ldots$ & 0.924 \\
\hline S 12 & 4 & 5.000 & 1.5 & 1.500 & 3.134 & $\ldots$ & 1.009 \\
\hline S 13 & 4 & 4.000 & 31.9 & 1.875 & 1.460 & $\ldots$ & 0.757 \\
\hline S 14 & 4 & 4.600 & 31.9 & 1.630 & 1.104 & $\ldots$ & 0.891 \\
\hline S 15 & 4 & 5.000 & 31.9 & 1.500 & 0.935 & $\cdots$ & 0.969 \\
\hline S 16 & 3 & 3.559 & 1.5 & 2.107 & 4.639 & $\ldots$ & 0.897 \\
\hline S 17 & 3 & 3.650 & 4.1 & 2.055 & 2.818 & $\ldots$ & 0.948 \\
\hline S 18 & 3 & 3.559 & 1.5 & 2.107 & 4.639 & $\sqrt{ }$ & 0.897 \\
\hline S 19 & 3 & 3.650 & 4.1 & 2.055 & 2.818 & $\sqrt{ }$ & 0.948 \\
\hline E 1 & $\ldots$ & $\ldots$ & 1.5 & $\ldots$ & $\ldots$ & $\ldots$ & 0.947 \\
\hline E 2 & $\ldots$ & $\ldots$ & 4.1 & $\ldots$ & $\ldots$ & $\ldots$ & 0.953 \\
\hline E 3 & $\ldots$ & $\ldots$ & 11.6 & $\ldots$ & $\ldots$ & $\ldots$ & 0.959 \\
\hline E 4 & $\ldots$ & $\ldots$ & 31.9 & $\ldots$ & $\ldots$ & $\ldots$ & 0.955 \\
\hline
\end{tabular}

\section{RESULTS AND DISCUSSION}

\section{A. Overview of simulations}

In Table II, an overview of the simulations is given. Cases $S 1-S 4$ were chosen as base settings for the simulations. In the following two sections, these simulations will be used to discuss various aspects of the flow field and sedimentation trajectory and will be compared to experimental results. In cases $S 5-S 8$, the input radius was varied between 2 and 8 grid nodes to study the influence of resolution on the accuracy and performance of the simulation. Simulations $S 9-S 15$ were done to study the sensitivity of the simulations to the hydrodynamic radius. Finally, in simulations $S 18$ and $S 19$, the lubrication force was applied to explore its usefulness for representing the bottom approach of the sphere. All simulations were executed for a physical time of approximately 4 seconds.

\section{B. Sedimentation trajectory}

In this paragraph, a physical interpretation of the sedimenting sphere is given based on the results of simulations $S 1$ and $S 4$, to demonstrate the characteristic differences in behavior of the particle sedimentation between both cases and to provide physical insight in the different flow cases. In Fig. 6 the flow field is plotted at four different stages during settling. In Fig. 7, the development of the kinetic energy of the sphere and the fluid is given. The intermediate results at Reynolds numbers of 4.1 and 11.6 have been left out.

The velocity field of the particle settling at $R e=1.5$ is not symmetric in the direction of motion [Fig. 6(a2)], dem- onstrating that the flow field is not in the Stokes regime. In Fig. 6(a1), the sphere has moved one diameter below the point of release. At this moment, the sphere has almost reached its steady-state velocity. The rate at which the flow field develops is demonstrated in Fig. 7(a), by the evolution of the kinetic energy of the fluid, which keeps pace with the kinetic energy of the sphere. As the sphere approaches the bottom, the kinetic energy of the wake again reduces in pace with the decay of kinetic energy of the particle, which decelerates because of the squeezing of the liquid between the sphere and the bottom wall [Fig. 6(a3)]. This causes the flow field in front of the sphere to deform and generates outward fluid motion across the bottom. Very quickly after the sphere has come to a halt, the fluid also comes to rest.

The particle settling at a Reynolds number of 31.9 shows distinctively different behavior. Compared to the case of $R e=1.5$ a much larger, elongated wake develops. In Fig. 6(b3), the deflected front of the flow field at bottom approach is again clearly visible. Another distinctive feature is that while both the kinetic energy of the particle and the fluid increase at the same pace initially, the particle reaches a plateau while the wake keeps on picking up kinetic energy until the particle reaches the tank bottom. At this stage, the kinetic energy of the fluid has not reached a steady state. Notice that the level of kinetic energy of both the fluid and the particle at the high Reynolds number are about one order of magnitude larger than that of the $R e=1.5$ case. Figure 7(b) further demonstrates that the sphere abruptly comes to a halt when the sphere hits the bottom wall, which is in contrast with the more gentle deceleration of the sphere at $R e=1.5$. After the 


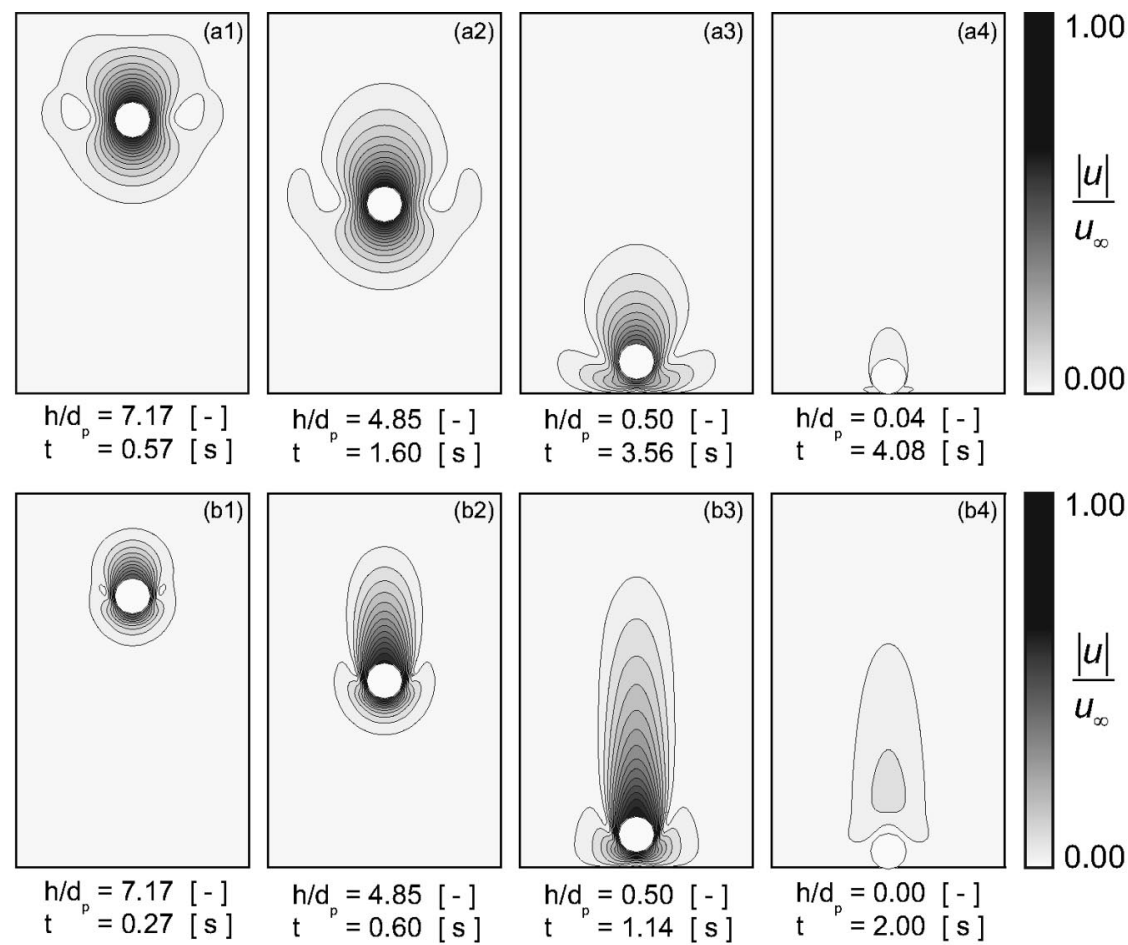

FIG. 6. Comparison of the flow field of the sedimenting sphere at (a), Re $=1.5$ (top, case $S 1$ ) and (b), Re $=31.9$ (bottom, case S4). The contours indicate the normalized velocity magnitude. $h / d_{p}$ indicates the dimensionless gap between the bottom apex of the sphere and the tank bottom wall, $t$ indicates time. sphere has come to a halt, the wake still contains a considerable amount of kinetic energy that slowly decays. An empirical time constant related to this process can be estimated by assuming an exponential function

$$
K_{f}(t)=K_{f, \max } \exp \left(\frac{-t}{\tau_{d}}\right),
$$

which is plotted in Fig. 7(b) with $\tau_{d}=0.3[\mathrm{~s}]$.

A remark can be made on the distribution of the kinetic energy over the particle and the fluid. During settling the potential energy of the particle is transferred to the fluid and dissipated. At maximum settling velocity, the kinetic energy of the fluid is much larger than that of the particle. The volume of the moving fluid is much larger than the volume of the particle (see also Fig. 6) and since the solid-liquid density ratio is small, the fluid can easily contain much more kinetic energy than the particle.
Three physical time scales can be used to interpret the flow field and transient behavior of the sedimenting sphere. The first time scale is the particle advection time $\left(\tau_{p, a}\right.$ $\simeq d_{p}\left(u_{\infty}\right)$, which is a measure for the time it takes the particle to travel one sphere diameter. The second time scale is the particle relaxation time, $\left(\tau_{p, r} \simeq \rho_{p} d_{p}^{2} / 18 \rho_{f} \nu\right)$ which is a measure for the time it takes for a particle to respond to an acceleration. The third time scale is the momentum diffusion time $\left(\tau_{\nu} \simeq d_{p}^{2} / \nu\right)$, which is a measure for diffusion of momentum into the fluid over a distance of one particle diameter. These three groups determine two independent dimensionless numbers, the Reynolds and Stokes number.

The different shapes of the flow field in the high and low Reynolds number cases can be interpreted by regarding the Reynolds numbers as the ratio of $\tau_{\nu}$ and $\tau_{p, a}$. At $R e=1.5$, $\tau_{\nu}$ is $0.59 \mathrm{~s}$ and $\tau_{p, a}$ is $0.39 \mathrm{~s}$. The time it takes for the particle to travel one diameter is almost the same as the time

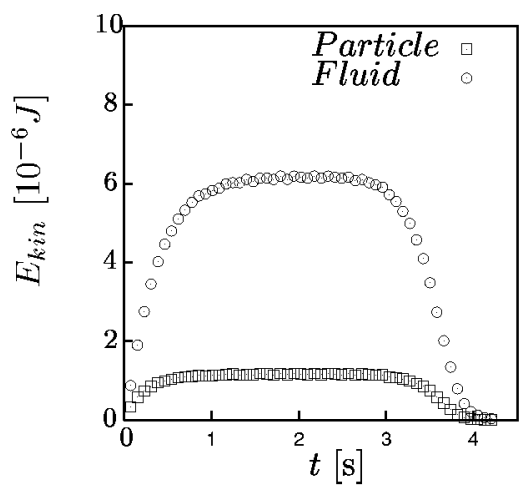

(a)

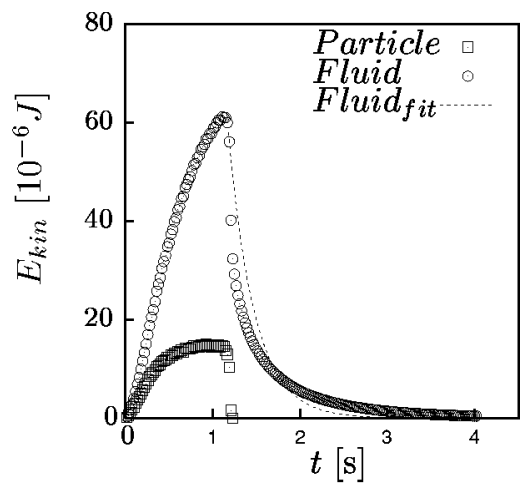

(b)
FIG. 7. Simulated result of the kinetic energy of sphere and fluid vs time at $R e=1.5$, case $S 1$, (a) and $R e$ $=31.9$, case $S 4$, (b). The dashed line in (b) is an exponential fit to the decay of the fluid kinetic energy. 


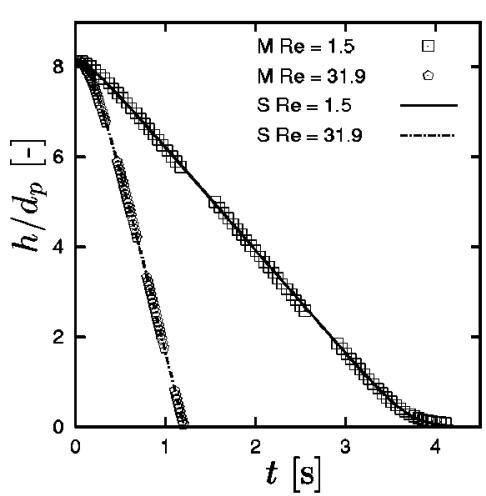

(a)

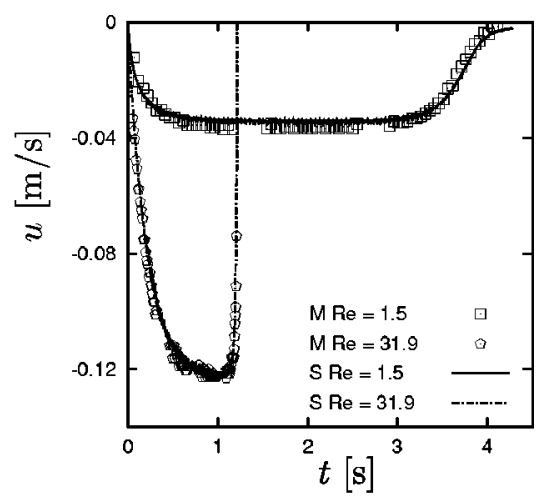

(b)
FIG. 8. Comparison between measured (M) and simulated (S) sphere trajectory (a), represented by the dimensionless gap height $h / d_{p}$, and sedimentation velocity (b) at two Reynolds numbers (simulation data from $S 1$ and $S 4)$. it takes for momentum to diffuse one diameter into the fluid. This explains the penetration of the flow field into the fluid in front of the sphere and sideways to the sphere over a length comparable to the size of the wake. In contrast to this, the shape of the wake at $R e=31.9$ is completely different. The characteristic time scales are $\tau_{\nu}=3.72 \mathrm{~s}$ and $\tau_{p, a}=0.12 \mathrm{~s}$. Thus, momentum diffusion goes at a much slower rate than particle advection, resulting in an elongated wake and a very limited extension of the flow field in front of the sphere.

The time scale for momentum diffusion can also be used to interpret Fig. 7. At $R e=1.5$, the diffusion time scale is much shorter than the time it takes for the particle to reach the bottom, thus allowing the wake to develop into a steady state. At $R e=31.9$, the particle reaches the bottom after approximately 1.3 seconds while $\tau_{\nu}$ is 3.72 seconds, which explains why the wake was not fully developed before the bottom was reached. It is interesting to note that the time constant for decay of the kinetic energy is much shorter than the time constant for momentum diffusion. The decay of kinetic energy is associated with the dissipation due to viscous effects that originate from gradients in the fluid. This process is apparently much faster than the momentum diffusion.
The second dimensionless number is the Stokes number $\left[S t=\left(\frac{1}{9}\right) \rho_{p} d_{p} u_{\infty} / \rho_{f} \nu=\left(\frac{1}{9}\right) \operatorname{Re} \rho_{p} / \rho_{f} \sim \tau_{p, r} / \tau_{p, a}\right]$, which is a measure for the ratio of particle inertia to viscous forces. With a practically constant density ratio throughout the experiment, the Stokes number is proportional to the Reynolds number and was not varied independently. The Stokes number characterizes the transient behavior of the particle at acceleration and bottom approach. At the low Reynolds number, the particle starts to decelerate at some distance from the bottom while at high Reynolds number, the particle hardly decelerates prior to contact.

\section{Comparison of numerical and experimental results}

A first comparison is made in terms of the maximum velocity of the particle during sedimentation. In Table II, the ratios $u_{\max } / u_{\infty}$ from the experiments and simulations are given. The experimental results demonstrate that the particle reaches a maximum velocity of approximately $95 \%$ of the steady-state value in an infinite medium. The experimental data indicate a maximum of the velocity ratio value for case $E 3$. An explanation for this observation is that the sphere (a) $\operatorname{Re}=1.5$
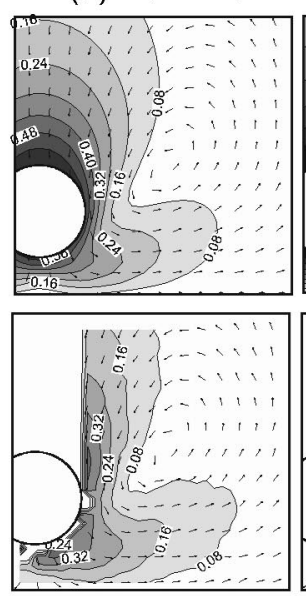

(b) $\operatorname{Re}=4.1$
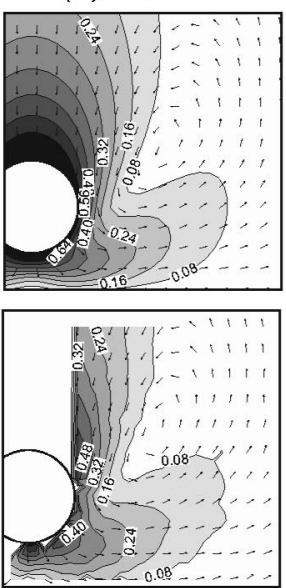

(c) $\operatorname{Re}=11.6$
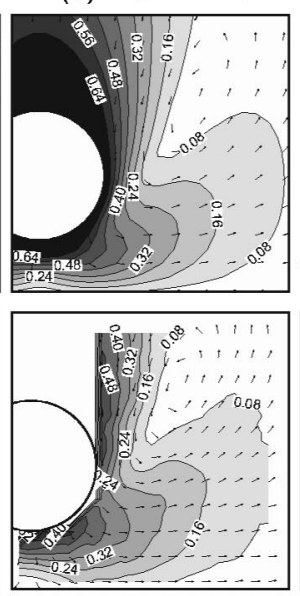

(d) $\operatorname{Re}=31.9$

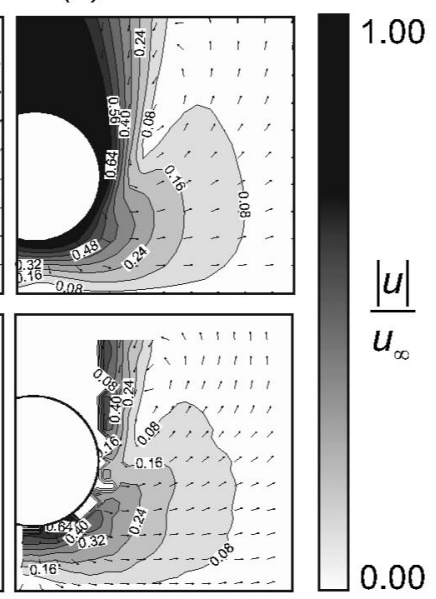

FIG. 9. Comparison of the simulated (top, cases $S 1-S 4$ ) and measured (bottom, $E 1-E 4$ ) flow field of the sphere at a dimensionless gap height of $h / d_{p}$ $=0.5$. Contours indicate the normalized velocity magnitude, the vectors indicate the direction of the fluid flow only. 


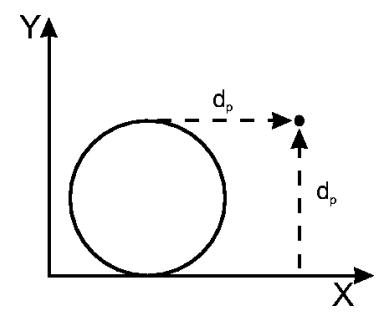

FIG. 10. Measurement position of the time series of fluid flow.

moving at the lowest Reynolds number experiences the largest resistance due to the container wall. At increasing Reynolds number, the lateral extension of the sphere induced flow reduces, thus reducing the wall hindrance effect on the sphere. The lower $u_{\max } / u_{\infty}$ ratio at the highest Reynolds number (i.e., case E4) is likely caused by the fact that the sphere was still accelerating when it arrived at the bottom. This trend is also observed in the simulated results, although more pronounced. The maximum sedimentation velocity predicted by the simulations is generally within $1 \%$ of the experimental result, except at the lowest Reynolds number, where the difference is approximately 5\%. Increased resolution does not result in an improvement. The calibration procedure has a strong impact on the terminal velocity. Its sensitivity will be further discussed in Sec. IV D.

For comparison of numerical and experimental findings as to the dynamic behavior of the sphere, the trajectory and velocity of the sphere versus time have been plotted in Figs. 8 (a) and 8(b). At the lowest Reynolds number, the sphere decelerates at a larger distance from the bottom than at the higher Reynolds number. Along with the results at $R e=4.1$ and $R e=11.6$ (not shown), these results demonstrate that the complete trajectory of the sphere is captured accurately by the simulation procedure.

The simulated fluid motion has been compared in detail with the flow fields from the PIV experiment. With the use of a continuous camera, not only the spatial structure of the flow field is obtained but also the temporal behavior. Figure 9 shows the flow field of the sphere at position $h / d_{p}=0.5$ at the four Reynolds numbers considered. [Notice that Figs. 9(c) and 9(d) are at a higher resolution than Figs. 9(a) and 9(b).] At the sphere position in question, the flow in front of the sphere interferes with the bottom surface, while the wake is still seemingly undisturbed. The correspondence in position of the velocity magnitude contours is indicative of a good agreement between the numerical and experimental flow field. At the side of the sphere a clear vortex is found that changes shape and position with an increase in Reynolds number. The center of this vortex is found at the same position for the numerical and experimental result.

Another (quantitative) assessment is obtained by comparing the time series of the fluid velocity in a particular point in the flow domain. As monitor point we chose a point fixed in place, positioned one diameter from the bottom of the tank and one diameter out of the center of the sphere (see Fig. 10).

Time series of the fluid velocity in this point are given for $R e=1.5$ (Fig. 11) and $R e=31.9$ (Fig. 12). At the low Reynolds number, the fluid flow in the $x$ direction is mainly effected by the squeezing action of the sphere. A distinctive positive peak is observed, due to the outward motion of the fluid. The fluid velocity drops almost back to zero at the moment the sphere touches the bottom of the container. At $R e=31.9$, the sphere settling velocity is much higher; as a result, the $x$ velocity starts rising much earlier in time. After the vortex has passed the monitor point, the velocity decreases again. As the sphere comes to rest, the fluid motion in the wake still contains a considerable amount of inertia and passes over the sphere, giving rise to the slight increase in $x$ velocity, followed by the decay to zero.

At $R e=1.5$, the flow in the $y$ direction is directed downwards, indicated by the negative value of the velocity component. The experimental data shows a steep decay to a minimum $y$ velocity, after which the velocity rises again.
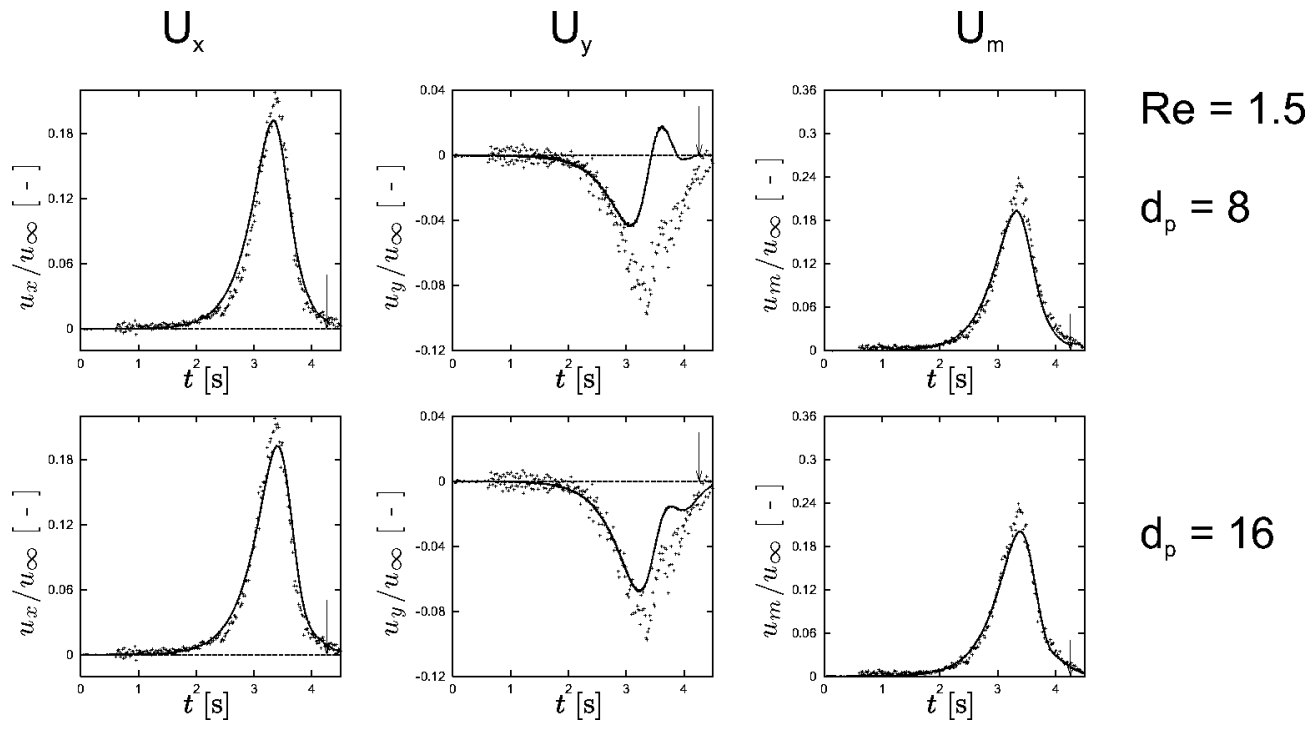

$$
d_{p}=16
$$

FIG. 11. Time series of the fluid velocity in a point at $R e=1.5$. The lines indicate the normalized velocity in $x$ and $y$ direction and velocity magnitude of simulations $S 1$ (top) and $S 5$ (bottom). The dots indicate the experimental result of $E 1$. The arrow indicates the moment the sphere comes to rest at the bottom. 

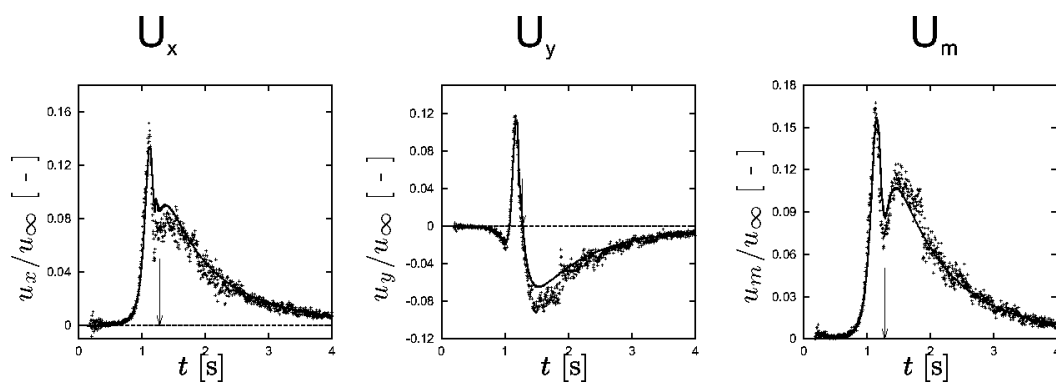

$\operatorname{Re}=31.9$
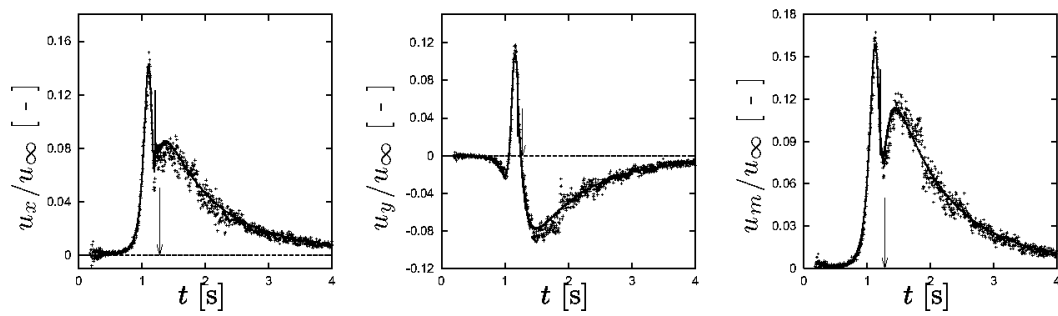

$d_{p}=16$

FIG. 12. Same as Fig. 11, now for $R e=31.9$.

With careful observation, a second, smaller decay can be observed in the data before returning to zero, although this decay is barely visible since the magnitude of the decay is comparable to the noise in the data. This behavior is related to both the position of the vortex center relative to the monitor point and the decreasing velocity of the sphere. At this Reynolds number the flow field extends the furthest sideways into the fluid and the monitor point is positioned between the sphere and the vortex center. As a result, the $y$ velocity does not change sign. Since the $y$ velocity is smaller near the core of the vortex, an increase in velocity may be found as the center passes the monitor point, after which a velocity decrease is anticipated. However, since the particle decelerates, the magnitude of this decrease is smaller than the main negative peak.

At $R e=31.9$ the monitor point is positioned on the right side of the vortex. Thus, the $y$ velocity initially gets negative, but as the vortex passes the monitor point, the $y$ velocity changes sign because the flow on the right side of the vortex center is directed upward. Eventually the vortex has passed and what follows is the wake of the sphere, resulting in a downward velocity that again slowly decays after the sphere has come to rest.

TABLE III. Influence of the hydrodynamic radius on the maximum sedimentation velocity.

\begin{tabular}{lccccc}
\hline \hline & $\begin{array}{c}r_{0} \\
{[\mathrm{lu}]}\end{array}$ & $\begin{array}{c}r_{h} \\
{[\mathrm{lu}]}\end{array}$ & $\begin{array}{c}R e \\
{[-]}\end{array}$ & $\begin{array}{c}u_{\max } \\
{\left[10^{-3} \mathrm{lu} / \mathrm{ts}\right]}\end{array}$ & $\begin{array}{c}u_{\infty} \\
{\left[10^{-3} \mathrm{lu} / \mathrm{ts}\right]}\end{array}$ \\
\hline Case & 4 & 4.000 & 1.5 & 7.678 & 10.000 \\
S 9 & 4 & 4.400 & 1.5 & 7.920 & 9.091 \\
S 10 & 4 & 4.487 & 1.5 & 7.968 & 8.914 \\
S 1 & 4 & 4.600 & 1.5 & 8.034 & 8.696 \\
S 11 & 4 & 5.000 & 1.5 & 8.074 & 8.000 \\
S 12 & 4 & 4.000 & 31.9 & 7.573 & 10.000 \\
S 13 & 4 & 4.600 & 31.9 & 7.749 & 8.696 \\
S 14 & 4 & 4.810 & 31.9 & 7.877 & 8.315 \\
S 4 & 4 & 5.000 & 31.9 & 7.754 & 8.000 \\
S 15 & 4 & & & &
\end{tabular}

At $R e=1.5$, the $y$ velocity shows the largest difference between simulation and experimental result. At low resolution of the simulation, a clear mismatch is observed between the numerical and experimental curve. Apparently, at lower Reynolds number, the position of the vortex is very sensitive to the resolution. The fluid velocity becomes positive, which is indicative of the monitor point being at the outer side of the vortex. As the resolution is increased, the position of the vortex is predicted more accurately and the simulated time series of the $y$ velocity is in much better agreement with the experimental result. The simulations at the high Reynolds number are in good agreement with the experimental data, although at this Reynolds number too, an increase in resolution improves the predictions. The curves that represent the velocity magnitude vs time demonstrate that at the low Reynolds number, the fluid velocity is underpredicted by a few percent only, which is in agreement with the contour plots of Fig. 9.

\section{Hydrodynamic radius dependency}

In simulations $S 9-S 15$, the hydrodynamic radius was varied deliberately (i.e., without applying the calibration procedure) to study its impact on $u_{\max } / u_{\infty}$. Without calibration the velocity ratio is underpredicted some $20 \%$. The simulations further show that the velocity ratio $u_{\max } / u_{\infty}$ is strongly dependent on the hydrodynamic radius. When varying the hydrodynamic radius on purpose, both $u_{\max }$ and $u_{\infty}$ change, as can be seen in Table III.

First, increasing the hydrodynamic radius may increase $u_{\max }$. The force $F_{d \text {,input }}$ drives the sphere during sedimentation and is determined from the balance between drag force, gravity and buoyancy

$$
F_{d, \text { input }}=\frac{4}{3} \pi r_{h}^{3}\left(\rho_{f}-\rho_{p}\right) g .
$$

When the sphere moves at $u_{\max }$, this force is balanced by the hydrodynamic forces that act on the sphere and which are obtained from the simulation. $F_{d \text {,input }}$ is independent of the 


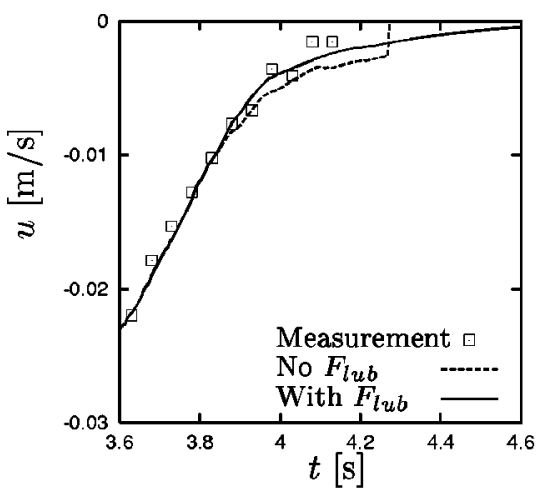

(a)

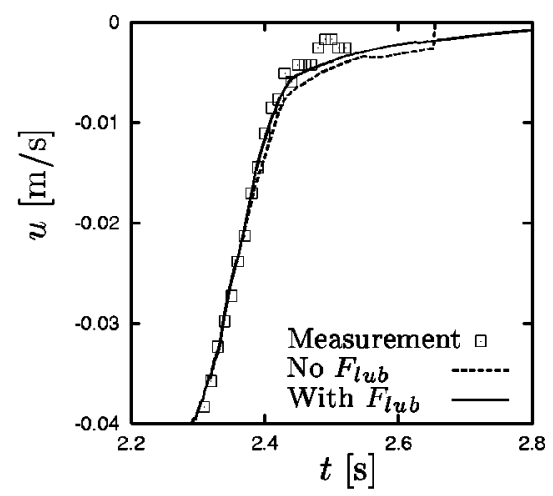

(b)
FIG. 13. The use of a lubrication force $\left(F_{\text {lub }}\right)$ on the particle sedimentation velocity at near wall approach at (a) $R e=1.5$, cases $S 16$ and $S 18$, and (b) $R e=4.1$, cases $S 17$ and $S 19$. hydrodynamic radius. With a changing hydrodynamic radius, length scales proportional to $L$, while time scales with the length squared, $L^{2}$, because time is scaled via the kinematic viscosity. Thus, when scaling Eq. (12) from physical quantities to simulation quantities, the equation is independent of the scaling factors, because $\rho$ is kept constant, $r_{h}^{3}$ scales as $L^{3}$ while $g$ scales as $1 / L^{3}$. At the same time, the input radius of the sphere and the viscosity are kept constant. Therefore, $u_{\max }$ can only vary because a change in the container geometry occurs. By increasing the hydrodynamic radius, the scaling factor for length decreases and the container geometry is represented by a larger number of grid nodes. Consequently, because of the larger domain, the sphere experiences a smaller resistance due to the parallel walls and $u_{\max }$ increases. The sensitivity of this effect depends on the Reynolds number at which sphere settles, as can be observed in Table III. At $R e=1.5, u_{\max }$ increases by $5.2 \%$ when varying the hydrodynamic radius from 4 to 5 grid units while at $R e$ $=31.9, u_{\max }$ increases by $2.4 \%$ only.

Second, when increasing the hydrodynamic radius, $u_{\infty}$ decreases as $1 / L$, which can be observed in Table III where $u_{\infty}$ is recalculated to lattice units. Thus, $u_{\infty}$ decreases by $20 \%$ for both the low and the high Reynolds number when increasing the hydrodynamic radius from 4 to 5 lattice units.

For the cases studied, these dependencies show that a variation in radius of the order of one lattice unit mainly affects the reference state $u_{\infty}$. In our base cases (with $r_{0}$ $=4 l u$ ), increasing the hydrodynamic radius with 1 lattice unit resulted in a variation of the ratio $u_{\max } / u_{\infty}$ of approximately $30 \%$. Increasing the resolution may decrease this sensitivity. A variation of one lattice unit on an input radius of, e.g., 8 lattice nodes causes $u_{\infty}$ to vary by $11 \%$ while the relative increase in $u_{\max }$ is expected to be smaller.

The calibration procedure proposed in Sec. III is used to determine the hydrodynamic radius a priori. Table II demonstrates that using this calibration method results in a $1 \%$ accurate match between the numerical and experimental values of the velocity ratio is found for the simulations at $R e$ $\geqslant 4$. . At the lowest Reynolds number, a systematic underprediction of the velocity ratio of approximately $5 \%$ is found. This difference corresponds to the deviations observed in Fig. 8. These deviations are considered acceptable, since without calibration an underprediction of more than $20 \%$ is obtained. However, the systematic deviation at the lowest Reynolds number is striking for two reasons. One, the deviation is independent of the resolution (see Table II). If the deviation decreases with an increase in resolution, one would anticipate the simulations to eventually match the experimental data at high resolution, which is not the case. Two, one would expect that the calibration procedure would work best at the lowest Reynolds number, since it is based on creeping flow conditions. The simulation at $R e=1.5$ comes closest to this situation.

\section{E. Lubrication force}

When the sphere approaches the bottom wall, the gap between the sphere and the bottom may become too narrow for a proper resolution of the flow on the original grid. As a result, the hydrodynamic force on the sphere will be underpredicted. In Fig. 13, the sedimentation velocities of the sphere at Reynolds numbers of 1.5 and 4.1, respectively, are given at the final stage of bottom approach. In the simulations, the particle velocity is set to zero at the moment of bottom contact. This moment is clearly visible in both figures, where the dotted line indicates the sedimentation velocity of the sphere in simulations $S 16$ and $S 17$. This abrupt stop indicates that the sphere velocity was not reduced to zero at the moment contact was established between the sphere and the bottom wall. The dotted line further shows unphysical fluctuations in the velocity of the sphere when the bottom of the sphere passes the first nodes above the bottom wall. Simulations at higher resolution showed that an increase in resolution reduces the fluctuations but the abrupt stop remains.

When applying the sub-grid scale lubrication force according to Eq. (10), the velocity of the sphere reduces more gradually although some fluctuations are still observed. The use of the lubrication force improves the velocity decay initially, which is demonstrated by an improved correspondence between the experimental and numerical data. Due to the dissipative action of the lubrication force, however, at a small separation from the bottom the settling velocity has almost reached zero (of the order of the numerical accuracy) and the sedimentation time series extends further for an unrealistically long time (not in the figure). Application of a force based on lubrication theory is valid for separations that exceed either the molecular mean free path length of the 
molecules of the fluid ${ }^{34}$ (although this effect is negligible for solid-liquid suspensions) or that exceed the surface roughness ${ }^{6}$ of the particle and the tank wall. For detailed simulations or experiments either of the two limits can be used as a cut-off measure for the final separation at which an apparent contact is established. In the current study, both effects are of the order of $0.1-1 \mu \mathrm{m}$ or smaller, which is of a much greater detail than provided by either our experimental observations or numerical simulations. The application of a lubrication force improves the simulation result in that it provides a measure for a lacking sub-grid scale repulsive force at bottom approach. At the same time it raises a difficulty in establishing the exact moment of contact between the particle and the container wall.

\section{CONCLUSION}

We investigated the motion of a single sphere settling in a box filled with silicon oil. By keeping the ratio of the sphere radius to the box dimensions relatively small, we were able to perform simulations of the full flow field without having to make specific assumptions on the external boundary conditions. We were able to validate the transient behavior of the sphere over the whole time span of the sedimentation from release via steady fall to deceleration at bottom approach. Time series of the particle trajectory and particle settling velocity were measured and detailed snapshots of the flow field were produced by using PIV. The data could also be represented as time series of the fluid velocity in monitor points. Lattice-Boltzmann simulations of cases identical to the experiments were performed. The boundary conditions for the solid sphere were imposed using the adaptive force field technique. This technique requires the sphere to have internal fluid that contributes to the sphere's inertia. A correction method has been proposed to compensate for this inertial effect. The simulations also require a correction for the hydrodynamic radius. The data demonstrated that the simulations are in agreement with measurements over a range of resolutions between 2 and 8 grid nodes per sphere radius. The transient behavior of both the sphere and fluid motion is captured accurately, as demonstrated by a comparison between experimental and numerical results in terms of particle trajectory and velocity as well as of fluid velocity. The hydrodynamic radius was found to affect the sedimentation velocity in two ways. First, a change in hydrodynamic radius causes a change in domain size, which varies the sedimentation velocity by $2 \%-5 \%$, depending on the Reynolds number. Second, a change in radius causes a change in time scaling, resulting in a variation of the velocity up to $20 \%$ for a sphere with an input radius of 4 lattice units. A calibration procedure was used for a priori determining the hydrodynamic radius of the sphere. For the cases of $R e=4.1$ to $R e$ $=31.9$, this calibration procedure predicts the maximum sedimentation velocity within $1 \%$ accuracy. At $R e=1.5$, the sedimentation velocity was underpredicted by approximately 5\% (independent of resolution). At approach of the bottom wall, resolution lacks to resolve the flow in the gap. Lubrication theory was used to provide the lacking hydrodynamic interactive force at bottom approach, but this apparently overpredicts the time to contact with the bottom.

\section{ACKNOWLEDGMENTS}

The authors like to thank Dr. L. Portela for the many fruitful discussions which contributed considerably to this work. We would like to thank Linvision for providing computational facilities. The authors also kindly thank Dr. J. Westerweel (Laboratory for Aero and Hydrodynamics, Delft University of Technology) for the use of the PIVWare software for the PIV flow field analysis.

${ }^{1}$ A. ten Cate, J. J. Derksen, H. J. M. Kramer, G. M. van Rosmalen, and H. E. A. van den Akker, "The microscopic modelling of hydrodynamics in industrial crystallisers," Chem. Eng. Sci. 56, 2495 (2001).

${ }^{2} \mathrm{H}$. Brenner, "The slow motion of a sphere through a viscous fluid towards a plane surface," Chem. Eng. Sci. 16, 242 (1961).

${ }^{3}$ P. Gondret, M. Lance, and L. Petit, "Bouncing motion of spherical particles in fluids," Phys. Fluids 14, 643 (2002).

${ }^{4}$ P. Gondret, E. Hallouin, M. Lance, and L. Petit, "Experiments on the motion of a solid sphere toward a wall: From viscous dissipation to elastohydrodynamic bouncing," Phys. Fluids 11, 2803 (1999).

${ }^{5}$ R. Zenit and M. L. Hunt, "Mechanics of immersed particle collisions," J. Fluids Eng. 121, 179 (1999).

${ }^{6}$ G. Joseph, R. Zenit, M. L. Hunt, and A. M. Rosenwinkel, "Particle-wall collisions in a viscous fluid," J. Fluid Mech. 433, 329 (2001).

${ }^{7}$ M. Raffel, C. Willert, and J. Kompenhans, Particle Image Velocimetry, A Practical Guide (Springer-Verlag, Heidelberg, 1998).

${ }^{8}$ A. J. C. Ladd, "Numerical simulations of particulate suspensions via a discretized Boltzmann equation. Part 1. Theoretical foundation," J. Fluid Mech. 271, 285 (1994).

${ }^{9}$ A. J. C. Ladd, "Numerical simulations of particulate suspensions via a discretized Boltzmann equation. Part 2. Numerical results," J. Fluid Mech. 271, 311 (1994).

${ }^{10} \mathrm{O}$. Behrend, "Solid-fluid boundaries in particle suspension simulations via the lattice Boltzmann method," Phys. Rev. E 52, 1164 (1995).

${ }^{11} \mathrm{D}$. Qi, "Lattice-Boltzmann simulations of particles in nonzero-Reynoldsnumber flows," J. Fluid Mech. 385, 41 (1999).

${ }^{12}$ C. K. Aidun, Y. Lu, and E.-J. Ding, "Direct analysis of particulate suspensions with inertia using the discrete Boltzmann equation," J. Fluid Mech. 373, 287 (1998).

${ }^{13}$ D. L. Koch and R. J. Hill, "Inertial effects in suspension and porousmedia flows," Annu. Rev. Fluid Mech. 33, 619 (2001).

${ }^{14}$ J. G. M. Eggels and J. A. Somers, "Numerical simulation of free convective flow using the lattice-Boltzmann scheme," Int. J. Heat Fluid Flow 16, 357 (1995).

${ }^{15}$ J. J. Derksen and H. E. A. Van den Akker, "Large eddy simulations on the flow driven by a Rushton turbine," AIChE J. 45, 209 (1999).

${ }^{16}$ J. J. Derksen and H. E. A. Van den Akker, "Simulation of vortex core precession in a reverse-flow cyclone," AIChE J. 46, 1317 (2000).

${ }^{17} \mathrm{~F}$. Abraham, "Functional dependence of drag coefficient of a sphere on Reynolds number," Phys. Fluids 13, 2194 (1970).

${ }^{18}$ J. Westerweel, "Digital particle image velocimetry-theory and application," Ph.D. thesis, Delft University of Technology, The Netherlands, 1993.

${ }^{19}$ Scilimage Install Guide, User's Manual, (Unix Version) (Technisch Physische Dienst, TNO-Delft University of Technology, Delft, 1994).

${ }^{20} \mathrm{~J}$. Westerweel, "Efficient detection of spurious vectors in particle image velocimetry data," Exp. Fluids 16, 236 (1994).

${ }^{21}$ S. Chen and G. D. Doolen, "Lattice Boltzmann method for fluid flows," Annu. Rev. Fluid Mech. 30, 329 (1998).

${ }^{22}$ R. Mei, L-S. Luo, and W. Shyy, "An accurate curved boundary treatment in the lattice Boltzmann method," J. Comput. Phys. 155, 307 (1999).

${ }^{23} \mathrm{H}$. Chen, C. Teixeira, and K. Molvig, "Realization of fluid boundary conditions via discrete Boltzmann dynamics," Int. J. Mod. Phys. C 9, 1281 (1998).

${ }^{24}$ R. Verberg and A. J. C. Ladd, "Lattice-Boltzmann model with sub-gridscale boundary conditions," Phys. Rev. Lett. 84, 2148 (2000).

${ }^{25}$ M. Rohde, J. J. Derksen, and H. E. A. Van den Akker, "Volumetric method 
for calculating the flow around moving objects in lattice-Boltzmann schemes," Phys. Rev. E 65, 056701 (2002).

${ }^{26}$ D. Goldstein, R. Handler, and L. Sirovich, "Modeling a no-slip flow boundary with external force field," J. Comput. Phys. 105, 354 (1993).

${ }^{27}$ C. K. Aidun and Y. Lu, "Lattice-Boltzmann simulation of solid particles," J. Stat. Phys. 81, 49 (1995).

${ }^{28} \mathrm{M}$. Heemels, "Computer simulations of colloidal suspensions using an improved lattice-Boltzmann scheme," Ph.D. thesis, Delft University of Technology, The Netherlands, 1999.

${ }^{29}$ X. He, Q. Zou, L-S. Luo, and M. Dembo, "Analytic solutions of simple flows and analysis of nonslip boundary conditions for the lattice Boltzmann BGK model,’ J. Stat. Phys. 87, 115 (1997).
${ }^{30} \mathrm{H}$. Hasimoto, "On the periodic fundamental solutions of the Stokes equations and their application to viscous flow past a cubic array of spheres," J. Fluid Mech. 5, 317 (1959).

${ }^{31}$ A. J. C. Ladd, "Sedimentation of homogeneous suspensions of nonBrownian spheres," Phys. Fluids 9, 491 (1997).

${ }^{32}$ C. Crowe, M. Sommerfeld, and Y. Tsuji, Multiphase Flows with Droplets and Particles (CRC, Boca Raton, 1997).

${ }^{33}$ S. Kim and S. J. Karrila, Microhydrodynamics: Principles and Selected Applications (Butterworth-Heinemann, Boston, 1991).

${ }^{34}$ R. R. Sundararajakumar and D. L. Koch, "Non-continuum lubrication flows between particles colliding in a gas," J. Fluid Mech. 313, 283 (1996) 